\title{
Evaluation of cardiac parameters between survivors and nonsurvivors of COVID-19 patients
}

\author{
Mehmet Ozdin'* (D), Hayrullah Yazar ${ }^{1,2}$ (1), Selcuk Yaylaci ${ }^{3}$ (D), Mehmet Koroglu ${ }^{4}$,

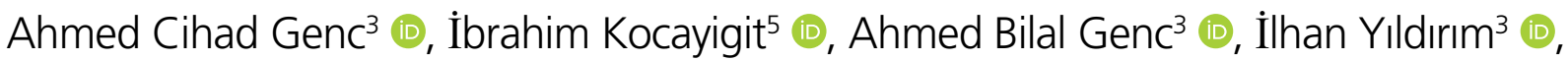 \\ Havva Kocayigit ${ }^{6}$ (1), Oguz Karabay ${ }^{7}$
}

\section{SUMMARY}

OBJECTIVE: The present study compares the cardiac parameters of the survivor and nonsurvivor patients with COVID-19 infection. METHODS: This study was conducted in 379 patients diagnosed with COVID-19 disease. Information of 21 nonsurvivor and 358 survivor patients with COVID-19 was obtained from the hospital information management system and analyzed retrospectively. Relationship between cardiac parameters in patients categorized into the mortal and immortal groups was investigated.

RESULTS: Of the total 379 patients involved in this study, 155 (40.9\%) were females and 224 (59.1\%) were males. No statistically significant difference in mortality was found between females and males $(p=0.249)$. The total median age was 70 , the median age in the nonsurvivor group was 74 (35-89), and it was 69.5 (18-96) in the survivor group $(p=0.249)$. The median values of high-sensitivity troponin (hs-Tn), creatine kinase MB form, and especially myoglobin in the survivor and nonsurvivor groups were 25/64.9 ( $p=0.028$ ), $18 / 23$ ( $p=0.02$ ), and 105.5/322.4 ( $p<0.001$ ), and the difference was statistically significant. Comparing mortality, while there was 1 $(0.7 \%)$ nonsurvivor out of 134 patients in the service unit, there were $20(8.2 \%)$ nonsurvivors out of 245 patients in the intensive care unit. This difference was statistically significant $(p=0.003)$. The cutoff value of myoglobin, which may pose a risk of mortality, was found to be $191.4 \mu \mathrm{g} / \mathrm{L}$, while it was $45.7 \mathrm{ng} / \mathrm{l}$ for hs-Tn and 60.1 U/L for creatine kinase MB.

CONCLUSIONS: Advanced age and increased levels of high-sensitivity troponin, creatine kinase MB, and myoglobin were found to be associated with mortality.

KEYWORDS: Troponin. Creatine kinase, MB form. Myoglobin. Mortality. Coronavirus infections.

\section{INTRODUCTION}

Coronaviruses cause severe acute respiratory syndrome (SARS) and were first appeared in 2002. The first zoonotic infection, which appeared in China in 2003, lead to SARS. The second coronavirus infection, Middle East Respiratory
Syndrome (MERS), was found in Saudi Arabia in 2012. The coronavirus disease 2019 (COVID-19), which first appeared in city of Wuhan, the capital of China's Hubei Province, has caused infections that have resulted in a pandemic with a significant impact on health and lives of people. It has first spread

\footnotetext{
'Sakarya University Education and Research Hospital, Medical Biochemistry Laboratory - Sakarya, Turkey.

${ }^{2}$ Sakarya University, Faculty of Medicine, Department of Medical Biochemistry - Sakarya, Turkey.

${ }^{3}$ Sakarya University, Faculty of Medicine, Department of Internal Medicine - Sakarya, Turkey.

${ }^{4}$ Sakarya University, Faculty of Medicine, Department of Medical Microbiology - Sakarya, Turkey.

${ }^{5}$ Sakarya University, Faculty of Medicine, Department of Cardiology - Sakarya, Turkey.

${ }^{6}$ Sakarya University, Faculty of Medicine, Department of Anesthesiology and Reanimation - Sakarya, Turkey.

${ }^{7}$ Sakarya University, Faculty of Medicine, Department of Infectious Disease and Clinical Microbiology - Sakarya, Turkey.

*Corresponding author: drmozdin33@gmail.com

Conflicts of interest: the authors declare there are no conflicts of interest. Funding: none.

Received on November 26, 2020. Accepted on November 29, 2020.
} 
in China, causing pneumonia-like disease. In January 2020, a novel SARS coronavirus (SARS-CoV-2, previously known as 2019-CoV), was reported by Chinese scientists linked to this disease ${ }^{1-3}$.

As with SARS and MERS coronaviruses, the SARS-CoV-2 spike (S) protein uses the angiotensin-converting enzyme 2 (ACE2) receptor for host cell entry ${ }^{4}$. The main target cells of the virus are type II pneumocytes and enterocytes, where ACE2 expression is high ${ }^{5}$. The $S$ protein binds to the catalytic domain of ACE2 with high affinity. Binding of the $S$ protein to ACE2 causes a conformational change in the $S$ protein of the coronavirus, allowing proteolytic digestion by host cell proteases, and thus viral RNA enters and infects the cell ${ }^{4,5}$.

COVID-19 infection spreads especially through droplets and direct contact among people, and is quite contagious. Although the incubation period is 5.5 days on average, it is known to last up to 14 days $^{6}$. Symptoms of COVID-19 are usually seen within the first 11-12 days of the disease. Fever, dry cough, fatigue, sore throat, and muscle pain are the most common symptoms in symptomatic cases. The additional, less-frequent symptoms include nausea/vomiting and diarrhea ${ }^{7}$. In_COVID19 patients, pneumonia is the primary indication for hospitalization by $91 \%$. Moreover, the causes of hospitalization include acute respiratory distress syndrome (ARDS) in 3.4\% of cases and shock in $1.1 \%$ of cases ${ }^{8}$. Some patients with COVID-19 have signs of cardiac damage. For this reason, the effects of the disease on the cardiovascular system have also been taken into account ${ }^{9}$. It has been reported that the increased mortality due to cardiac damage observed in COVID-19 patients is also caused by the infection ${ }^{10}$.

Myoglobin is a globular protein molecule consisting of 153 amino acids. It is a single-chain protein containing hem group at the center and has a molecular weight of $16,700 \mathrm{Da}^{11}$. It is present in the heart and skeletal muscle. In addition to its oxygen storage task, myoglobin transfers oxygen to muscle cells ${ }^{12,13}$. It is not specific to the heart, but it is released earlier than creatine kinase $\mathrm{MB}$ form (CK-MB) in the necrotic myocardium and increases in circulation within $2 \mathrm{~h}$. Its elevation in the serum lasts about $24 \mathrm{~h}^{14}$.

Troponins (hs-Tn) are found in skeletal and cardiac muscle structures together with tropomyosin. Troponins are structural proteins involved in regulating skeletal and cardiac muscle contraction ${ }^{15}$. They are sensitive and specific markers for the damage in the heart muscle and were accepted as the standard marker in the year 2000 by the European Society of Cardiology/ American College of Cardiology (ESC/ACC) in the diagnosis of acute myocardial infarction (MI), and by the ACC/American Heart Association (AHA) in the diagnosis and monitoring of unstable angina pectoris ${ }^{16-18}$.
Creatine kinase (CK) is a dimeric enzyme consisting of two subunits of $40 \mathrm{kDa}$ each. The $\mathrm{CK}$ measurements are mainly used in the diagnosis, follow-up, and in the treatment of MI. This enzyme is especially the most sensitive indicator of muscle damage. Although CK-MB has different levels in the heart muscle, its levels are very low in the skeletal muscle ${ }^{19}$.

In their renewed criteria to classify acute coronary syndromes in 2000, the European Society of Cardiology and the American Society of Cardiology ${ }^{20}$ proposed the cardiac troponin and CK-MB mass measurement as biochemical markers in the diagnosis of myocardial necrosis.

Since the COVID-19 pandemic is a quite recent phenomenon, studies on the characteristics and treatment of the virus and the disease are yet to be well established in the literature. Despite the numerous scientific studies published gradually in the literature, there is no adequate and accurate information about COVID-19 infection and its treatment. Considering the pathogenesis of the disease, manifestations, and test results in patients, the cardiac parameters/tests seem to be skewed/affected. There are only few studies investigating the cardiac parameters in patients with COVID-19 infection. Hence, this study aims to comparatively investigate the cardiac parameters of survivor and nonsurvivor COVID-19 patients.

\section{METHODS}

This study was approved by the Sakarya University Medical Faculty Ethics Committee (20.05.2020/ $\mathrm{N}^{\circ}$ : E.4618). A total of 379 patients diagnosed with COVID-19 disease and hospitalized in the service unit were included in this study. Information about these 21 nonsurvivor and 358 survivor patients was obtained from the hospital information management system and analyzed retrospectively. COVID-19 was diagnosed using clinical findings, computed tomography, and SARS-CoV-2 reverse transcriptase polymerase chain reaction (RT-PCR).

The hs-Tn, CK-MB, and myoglobin parameters, which play an important role in cardiac assessment, were evaluated on the first day of hospitalization.

\section{Cardiac parameters}

Myoglobin and hs-Tn tests were studied using the Architect i2000 SR immunoassay analyzer (Abbott, USA), and CK-MB tests were studied using the Beckman Coulter AU5800 autoanalyzer (Beckman-Coulter, USA).

\section{Statistical analysis}

The descriptive analyses were performed to provide information on general characteristics of the study population. Visual (probability plots and histograms) and analytical 
methods (Kolmogorov-Smirnov/Shapiro-Wilk test) were used to determine the normal distribution. The descriptive analyses were presented using medians and interquartile range (IR) for the non-normally distributed variables. The MannWhitney $U$ test was used for nonparametric tests to compare these parameters. The Pearson's $\chi^{2}$ test used to compare the categorical variables between two groups. The categorical variables were presented as the frequency (\%). A $p<0.05$ was considered significant. Analyses were performed using SPSS statistical software (IBM SPSS Statistics, Version 22.0, IBM Corp., Armonk, NY, USA).

\section{RESULTS}

Out of 379 patients, 155 (40.9\%) were females and 224 (59.1\%) were males with no statistically significant difference in mortality $(\mathrm{p}=0.249)$. The total median age range and IR was: 70 (18-96) [18]. The median age in the nonsurvivor group was $74(35-89)$ [16] and was 69.5 (18-96) [19] in the survivor group ( $\mathrm{p}=0.249$ ). The median values of hs-Tn, CK-MB, and especially myoglobin in the survivor and nonsurvivor groups, range, IR with $\mathrm{p}$ values were: $25(0.2-24,141.7)$ [79.85], $64.9(8-30,515)$ [1889.85], $\mathrm{p}=0.028 ; 18(5-218)$ [10.25], 23 (9-91) [16.85], $\mathrm{p}=0.02$; and 105.5 (13.9-1188.4) [166.35], 322.4 (35.6-941) [344.9], $\mathrm{p}<0.001$; and the difference was statistically significant. Comparing mortality, while there was $1(0.7 \%)$ nonsurvivor out of 134 patients in the service unit, there were $20(8.2 \%)$ nonsurvivor out of 245 patients in the intensive care unit. This difference was found to be statistically significant $(\mathrm{p}=0.003)$ (Table 1$)$. The receiver operating characteristic (ROC) analysis was performed for the cutoff values of myoglobin, hs-Tn, and CK-MB in COVID-19 patients at our center. The area under the ROC curve (AUC), cutoff value, sensitivity, and specificity were calculated. The cutoff value of myoglobin, which may pose a risk of mortality, was found to be $191.4 \mu \mathrm{g} / \mathrm{l}$ (AUC 0.732; 95\%CI 0.621-0.843; sensitivity 71.4; specificity 70.7; $\mathrm{p}<0.001$ ). It was $45.7 \mathrm{ng} / \mathrm{l}$ for hs-Tn (AUC 0.643; 95\%CI 0.529-0.756; sensitivity 57.1; specificity 65.6; $\mathrm{p}=0.028$ ) and 60.1 U/L for CK-MB (AUC 0.651 ; $95 \%$ CI $0.53-0.772$; sensitivity 61.9 ; specificity 60.1 ; $\mathrm{p}=0.02$ ) (Figure 1 and Table 2).

\section{DISCUSSION}

COVID-19 infection can affect the respiratory tract, causing a wide range of clinical manifestations ranging from viral pneumonia, which leads to severe respiratory failure, to mortality ${ }^{21}$. According to the pathogenesis of the COVID-19 disease, it affects the respiratory tract and cardiovascular system, leading to noticeable abnormalities in some blood parameters related to the organs affected. In this study, hs-Tn, CK-MB, and myoglobin parameters were investigated in survivor and nonsurvivor COVID-19 patients.

According to the studies conducted so far, although individuals in the above-middle age group were found to be severely affected by COVID-19, the rate of severity of the disease is higher in individuals of advanced age $\mathrm{e}^{22}$. The mortality risk is high when the elderly people with cardiovascular disease encounter the COVID-19 virus. The characteristics of the first 3200 patients who lost their lives in Italy, which initially

Table 1. Study parameters between survivor and nonsurvivors.

\begin{tabular}{|c|c|c|c|c|c|}
\hline & Total $(n=379)$ & Survivor $(n=358)$ & $\begin{array}{c}\text { Nonsurvivor } \\
(n=21)\end{array}$ & $p$ \\
\hline \multicolumn{2}{|c|}{ Age (median, range, [IR]) } & $70(18-96)[18]$ & $69.5(18-96)[19]$ & $74(35-89)[16]$ & $0.249^{a}$ \\
\hline \multirow{2}{*}{ Gender } & Female, n (\%) & $155(40.9)$ & $147(94.8)$ & $8(5.2)$ & \multirow{2}{*}{$0.788^{b}$} \\
\hline & Male, n (\%) & $224(59.1)$ & $211(94.2)$ & $13(5.8)$ & \\
\hline \multirow{2}{*}{ Hospitalization } & Ward, n(\%) & $134(35.4)$ & $133(99.3)$ & $1(0.7)$ & \multirow{2}{*}{$0.003^{b}$} \\
\hline & ICU, n (\%) & $245(64.6)$ & $225(91.8)$ & $20(8.2)$ & \\
\hline \multicolumn{2}{|c|}{ hs-Tn (median, range, [IR]) } & $\begin{array}{c}25.2(0.2-30,515.4) \\
{[81.1]}\end{array}$ & $\begin{array}{c}25(0.2-24,141.7) \\
{[79.85]}\end{array}$ & $\begin{array}{c}64.9(8-30,515) \\
{[1889.85]}\end{array}$ & $0.028^{a}$ \\
\hline \multicolumn{2}{|c|}{ CK-MB (median, range, [IR]) } & $18(5-218)[10.2]$ & $18(5-218)$ [10.25] & 23 (9-91) [16.85] & $0.02^{\mathrm{a}}$ \\
\hline \multicolumn{2}{|c|}{ Myoglobin (median, range, [IR]) } & $\begin{array}{c}112.1(13.9-1188.4) \\
{[175]}\end{array}$ & $\begin{array}{c}105.5(13.9-1,188.4) \\
{[166.35]}\end{array}$ & $\begin{array}{c}322.4(35.6-941) \\
{[344.9]}\end{array}$ & $0.000^{\mathrm{a}}$ \\
\hline
\end{tabular}

aMann-Whitney $U$ test; 'Pearson's chi-square test. Nonparametric values are expressed as median value, range values (min-max); interquartile range [IR]; hs-Tn, troponins; CK-MB, creatine kinase MB form. A p<0.05 was considered statistically significant. 


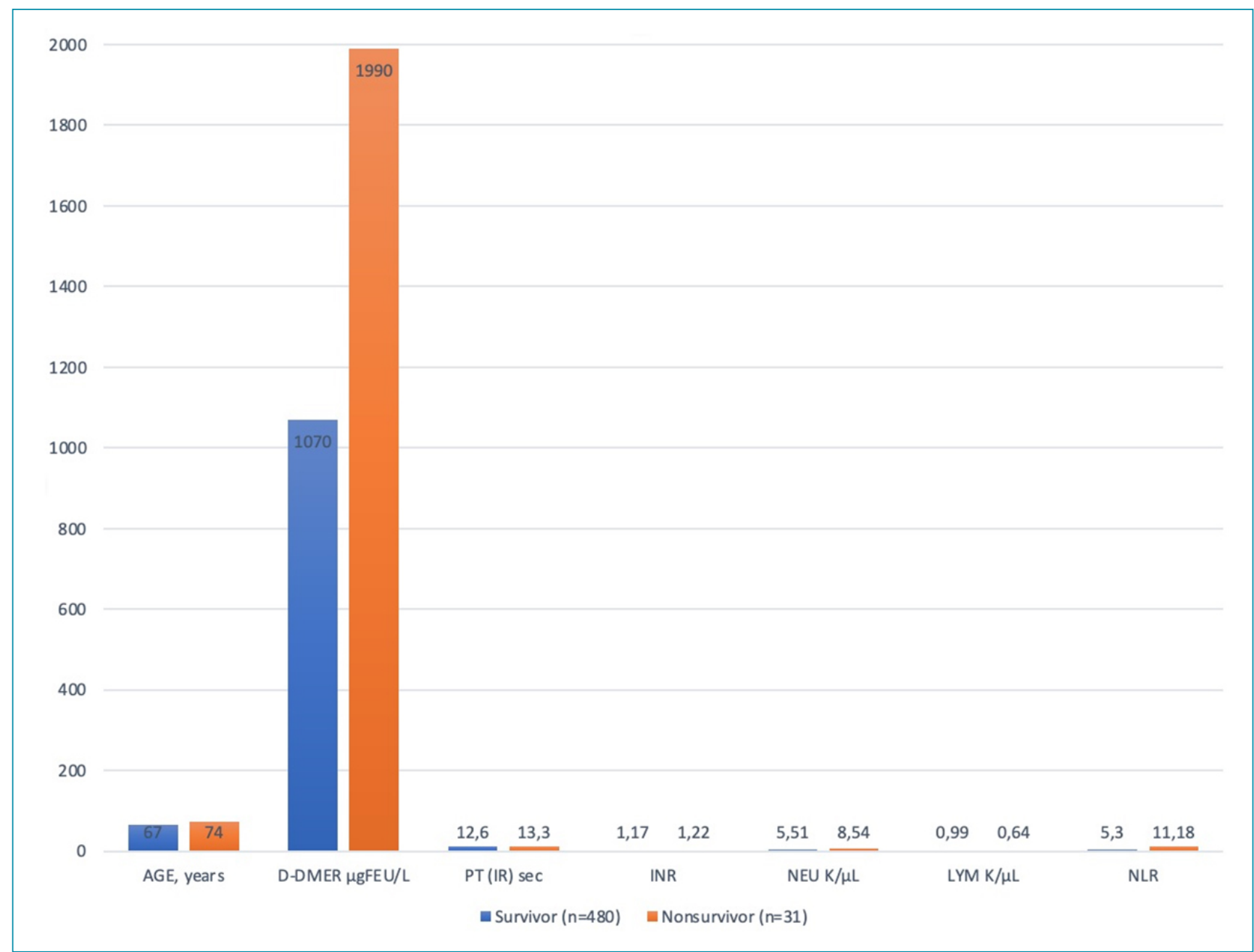

Figure 1. Receiver operating characteristic analysis chart for cutoff values of cardiac parameters to predict mortality risk in COVID-19 patients.

Table 2. Results of Receiver operating characteristic analysis to predict mortality in COVID-19 patients.

\begin{tabular}{l|c|c|c|c|c}
\hline Risk factor & AUC $(95 \% \mathrm{Cl})$ & Cutoff & Sensitivity (\%) & Specificity (\%) & $p$ \\
\hline Myoglobin & $0.732(0.621-0.843)$ & 191.4 & 71.4 & 70.7 & 0.00 \\
\hline hs-Tn & $0.643(0.529-0.756)$ & 45.7 & 57.1 & 65.6 & 0.028 \\
\hline CK-MB & $0.651(0.53-0.772)$ & 19.85 & 61.9 & 60.1 & 0.02 \\
\hline
\end{tabular}

AUC: area under curve; 95\%Cl: confidence interval; hs-Tn, troponins; CK-MB: creatine kinase MB form.

had the highest rate of mortality worldwide due to COVID19 , were investigated in a study. As a result, the average age of nonsurvivor patients due to COVID-19 infection was found to be 78.5 (31-103, IR 73-85 years $)^{23}$.

In the present study, it was found that the COVID-19 patients were above the middle age and the nonsurvivor patients were of advanced age with an average age of 74 .
The high-sensitivity cardiac hs-Tn levels are reported to be high in a significant proportion of COVID-19 patients. In a retrospective analysis study of 191 cases hospitalized due to COVID-19 infection, hs-cTnI levels were found to be $>50 \%$ in nonsurvivor patients. Typically, the hs-Tn levels increase gradually within days in nonsurvivor patients. The troponin increase continues on the 16th day and after 
until death. While the rate of cases was $46 \%$ among the nonsurvivor patients with hs-TnI $>28 \mathrm{pg} / \mathrm{mL}$, it was reported to be $1 \%$ in the survived patients $(\mathrm{p}<0.0001)$. In one-way analyses, a significant relationship was found between increased hs-Tn levels and mortality $(\mathrm{p}<0.0001)$. It is noted that a gradually increasing hs-Tn level day-by-day in COVID-19 patients is an important indicator of mortality ${ }^{21}$. A study of hs-Tn levels in severe COVID-19 patients found that the levels were significantly higher compared to cases with nonsevere infection ${ }^{24}$. Serum troponin-I levels that were high at the time of diagnosis or increased during follow-up were identified as poor prognostic factors associated with severity of the disease and mortality ${ }^{21}$. In a study of 273 COVID-19 patients with critical, severe, and mild cases, troponin levels of critical and severe cases were found to be statistically significantly different compared to mild cases $(\mathrm{p}<0.05)^{25}$. In this study, a statistically significant difference was found in the hs-Tn levels between survivor and nonsurvivor COVID-19 patients $(\mathrm{p}<0.028)$.

In a study of 273 COVID-19 patients categorized into critical, severe, and mild cases, statistically significant results were obtained in the myoglobin levels of critical and severe cases compared with mild cases $(\mathrm{p}<0.05)^{25}$. A study of COVID-19 patients found high levels of myoglobin in those patients ${ }^{26}$. In this study on COVID-19 patients, the difference in the myoglobin levels between survivor and nonsurvivor patients was found to be statistically significant $(\mathrm{p}<0.000)$.

In a study conducted in patients diagnosed with COVID19, CK-MB levels, which are among the cardiac markers, were found to be high ${ }^{3}$. In a study of 273 COVID-19 patients categorized into critical, severe, and mild cases, statistically significant results were obtained comparing CK-MB levels of critical and severe cases with mild cases $(\mathrm{p}<0.05)^{25}$. In this study on COVID-19 patients, a statistically significant difference was found in the CK-MB levels between survivor and nonsurvivor patients $(\mathrm{p}<0.02)$.

In our study, the cutoff value of myoglobin, which may pose a risk of mortality, was found to be $191.4 \mu \mathrm{g} / \mathrm{l}$, while it was $45.7 \mathrm{ng} / \mathrm{l}$ for hs-Tn and 60.1 U/L for CK-MB.

\section{CONCLUSION}

Advanced age and increased levels of hs-Tn, CK-MB, and myoglobin were found to be associated with mortality in COVID-19 patients.

\section{AUTHORS" CONTRIBUTIONS}

MO: Data Curation, Formal Analysis, Writing - Original Draft, Writing - Review \& Editing. HY: Data Curation, Formal Analysis, Writing - Original Draft, Writing - Review \& Editing. SY: Data Curation, Formal Analysis, Writing - Original Draft, Writing - Review \& Editing. MK: Data Curation, Formal Analysis, Writing - Original Draft, Writing-Review \& Editing. ACG: Conceptualization, Data Curation, Formal Analysis, Writing - Review \& Editing. IK: Conceptualization, Data Curation, Formal Analysis, Writing - Review \& Editing. ABG: Conceptualization, Data Curation, Formal Analysis, Writing - Review \& Editing. IY: Data Curation, Formal Analysis, Writing - Review \& Editing. HK: Data Curation, Formal Analysis, Writing - Review \& Editing. OK: Data Curation, Formal Analysis, Writing - Review \& Editing.

\section{REFERENCES}

1. Phelan AL, Katz R, Gostin LO. The novel coronavirus originating in Wuhan, China: challenges for global health governance. JAMA. 2020;323(8):709-10. https://doi.org/10.1001/jama.2020.1097

2. Gorbalenya AE, Baker SC, Baric RS, de Groot RJ, Drosten C, Gulyaeva AA, et al. Severe acute respiratory syndrome-related coronavirus: the species and its viruses - a statement of the Coronavirus Study Group. bioRxiv 2020; published online Feb 11. https://doi.org/10.1101/2020.02.07.937862

3. Yazar H, Kayacan Y, Ozdin M. De Ritis ratio and biochemical parameters in COVID-19 patients. Arch Physiol Biochem. 2020;1-5. https://doi.org/10.1080/13813455.2020.1788604

4. Li W, Moore MJ, Vasilieva N, Sui J, Wong SK, Berne MA, et al. Angiotensin-converting enzyme 2 is a functional receptor for the SARS coronavirus. Nature. 2003;426(6965):450-4. https:// doi.org/10.1038/nature02145
5. Hamming I, Timens W, Bulthuis ML, Lely AT, Navis G, van Goor $\mathrm{H}$. Tissue distribution of ACE2 protein, the functional receptor for SARS coronavirus. A first step in understanding SARS pathogenesis. J Pathol. 2004;203(2):631-7. https://doi. org/10.1002/path.1570

6. Lauer SA, Grantz KH, Bi Q, Jones FK, Zheng Q, Meredith $H R$, et al. The Incubation period of coronavirus disease 2019 (COVID-19) from publicly reported confirmed cases: estimation and application. Ann Intern Med. 2020;172(9):577-82. https:// doi.org/10.7326/M20-0504

7. Guan WJ, Ni ZY, Hu Y, Liang WH, Ou CQ, He JX, et al. China Medical Treatment Expert Group for Covid-19. Clinical characteristics of coronavirus disease 2019 in China. N Engl J Med. 2020;382(18):1708-20. https://doi.org/10.1056/ NEJMoa2002032 
8. Andersen KG, Rambaut A, Lipkin WI, Holmes EC, Garry RF. The proximal origin of SARS-CoV-2. Nat Med. 2020;26(4):450-2. https://doi.org/10.1038/s41591-020-0820-9

9. Huang $C$, Wang $Y$, Li $X$, Ren L, Zhao J, Hu Y, et al. Clinical features of patients infected with 2019 novel coronavirus in Wuhan, China. Lancet. 2020;395(10223):497-506. https:// doi.org/10.1016/S0140-6736(20)30183-5

10. Shi S, Qin M, Shen B, Cai Y, Liu T, Yang F, et al. Association of cardiac Injury with mortality in hospitalized patients with COVID-19 in Wuhan, China. JAMA Cardiol. 2020;5(7):80210. https://doi.org/10.1001/jamacardio.2020.0950

11. Ordway GA, Garry DJ. Myoglobin: an essential hemoprotein in striated muscle. J Exp Biol. 2004;207(Pt 20):3441-6. https:// doi.org/10.1242/jeb.01172

12. Vaidya H. Myoglobin. Lab Medicine [Internet]. 1992 [cited on Sept. 29, 2020];23(5):306-10. Available from: https://www. ilexmedical.com/files/PDF/Myoglobin_AXS.pdf

13. Bhayana $V$, Henderson AR. Biochemical markers of myocardial damage. Clin Biochem. 1995;28(1):1-29. https://doi. org/10.1016/0009-9120(94)00065-4

14. Kontos MC, Anderson FP, Schmidt KA, Ornato JP, Tatum JL, Jesse $\mathrm{RL}$. Early diagnosis of acute myocardial infarction in patients without ST-segment elevation. Am J Cardiol. 1999;83(2):155-8. https://doi.org/10.1016/s0002-9149(98)00816-9

15. Avcıküçük M, Bakır F, Topçuoğlu C, Güçtekin A. Akut koroner sendromda troponin T ve troponin I. Turk Hij Den Biyol Derg. 2011;68(3):127-34. https://doi.org/10.5505/ TurkHijyen.2011.85047

16. Sheehan P, Blennerhassett J, Vasikaran SD. Decision limit for troponin I and assay performance. Ann Clin Biochem. 2002;39(Pt 3):231-6. https://doi.org/10.1258/0004563021902161

17. Wu AH. Increased troponin in patients with sepsis and septic shock: myocardial necrosis or reversible myocardial depression? Intensive Care Med. 2001;27(6):959-61. https:// doi.org/10.1007/s001340100970

18. Morow DA, Rifai N, Tanasijevic MJ, Wybenga DR, Lemos JA, Antman EM. Clinical efficacy of three assays for cardiac troponin I for risk stratification in acute coronary syndrome: a Thrombolysis In Myocardial Infarction (TIMI) 11B Substudy. Clin Chem. 2000;46(4):453-60. PMID: 10759468
19. Alpert JS, Thygesen K, Antman E, Bassand JP. Myocardial infarction redefined: a consensus document of The Joint European Society of Cardiology/American College of Cardiology Committee for the redefinition of myocardial infarction. J Am Coll Cardiol. 2000;36(3):959-69. https://doi.org/10.1016/ s0735-1097(00)00804-4

20. Myocardial infarction redefined--a consensus document of The Joint European Society of Cardiology/American College of Cardiology Committee for the redefinition of myocardial infarction. Eur Heart J. 2000;21(18):1502-13. https://doi. org/10.1053/euhj.2000.2305

21. Zhou F, Yu T, Du R, Fan G, Liu Y, Liu Z, et al. Clinical course and risk factors for mortality of adult inpatients with COVID-19 in Wuhan, China: a retrospective cohort study. Lancet. 2020;395(10229):1054-62. PMID: 32171076

22. Mclntosh MK. Coronavirus disease 2019 (COVID-19): epidemiology, virology and prevention. UpToDate 2020 [07.05.2020]. [cited on May 7, 2020]. Available from: https:// www.uptodate.com/contents/coronavirus-disease-2019covid-19-epidemiolog virology-clinical-features-diagnosisand-prevention\#H4141359801

23. Palmieri L, Andrianou X, Bella A, Bellino S, Boros S, Canevelli $\mathrm{M}$, et al. Characteristics of COVID-19 patients dying in Italy Report based on available data on March 20th, 2020. COVID-19 Surveillance Group. [cited on Mar. 23,2020]. Available from: https://www.epicentro.iss.it/coronavirus/bollettino/ReportCOVID-2019_20_marzo_eng.pdf

24. Lippi G, Lavie CJ, Sanchis-Gomar F. Cardiac troponin I in patients with coronavirus disease 2019 (COVID-19): evidence from a meta-analysis. Prog Cardiovasc Dis. 2020;63(3):390-1. https://doi.org/10.1016/j.pcad.2020.03.001

25. Han H, Xie L, Liu R, Yang J, Liu F, Wu K. Analysis of heart injury laboratory parameters in 273 COVID-19 patients in one hospital in Wuhan, China. J Med Virol. 2020;92(7):819-23. https://doi.org/10.1002/jmv.25809

26. Li L, Zhou Q, Xu J. Changes of laboratory cardiac markers and mechanisms of cardiac injury in coronavirus disease 2019. Biomed Res Int. 2020;2020:7413673. https://doi. org/10.1155/2020/7413673 


\section{ERRATUM}

https://doi.org/10.1590/1806-9282.67.Suppl1.20200848ERRATUM

In the manuscript "Evaluation of cardiac parameters between survivors and nonsurvivors of COVID-19 patients", https://doi. org/10.1590/1806-9282.67.Suppl1.20200848, published in the Rev Assoc Med Bras. 2021;67(Suppl 1):80-5, on page 83 Figure 1:

Where it reads:

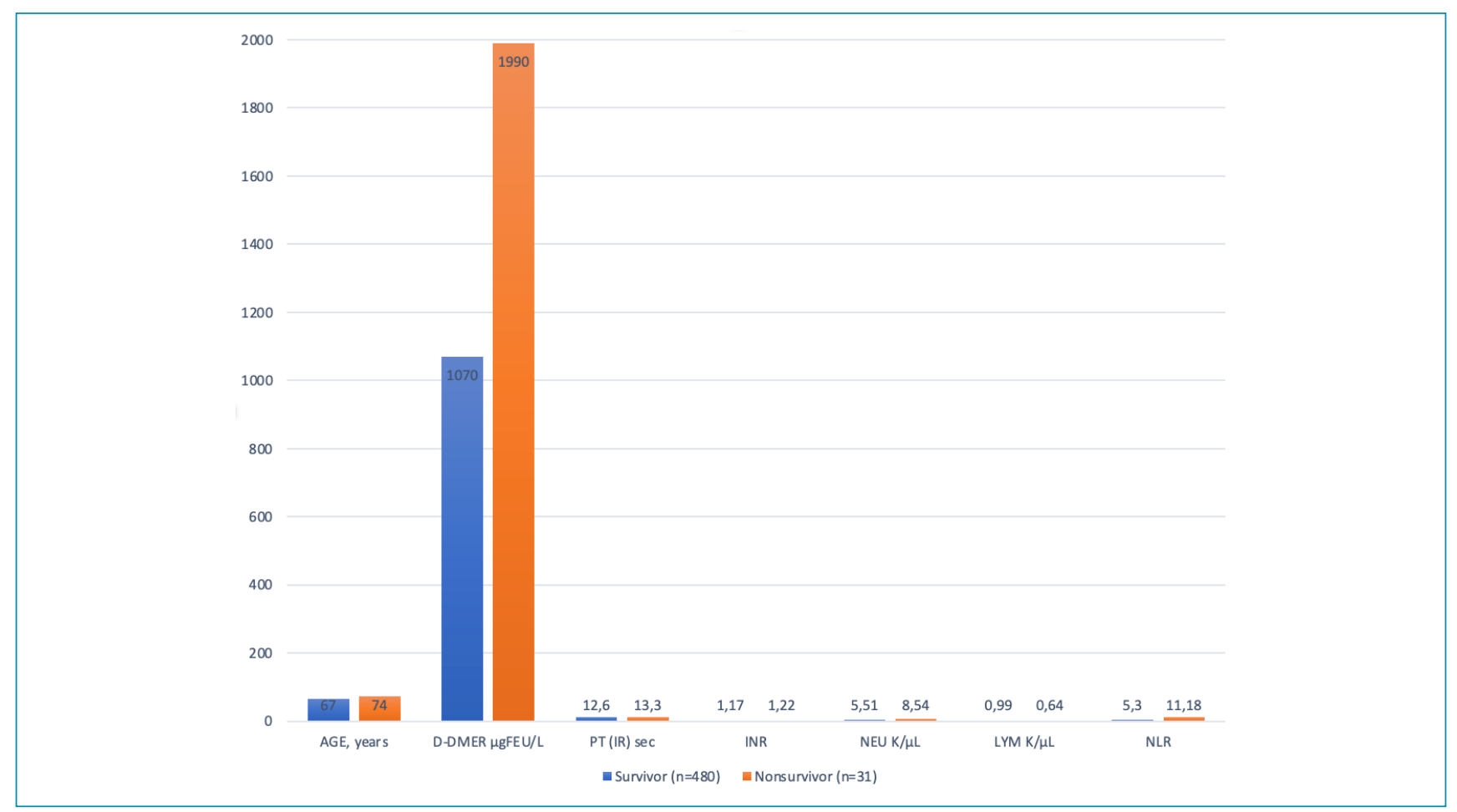

It should read:

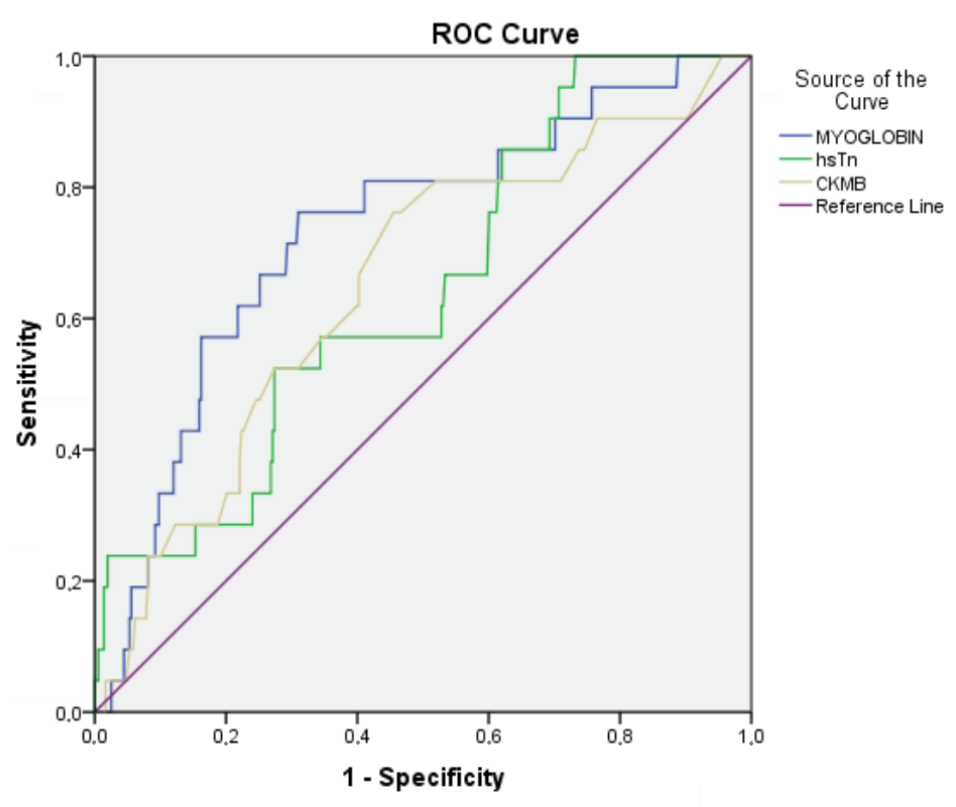

ii 